\title{
Commentary
}

Neuropsychobiology

Published online: December 19, 2017

\section{Systems Neuroscience of Psychosis (SyNoPsis) Provides a Promising Framework for Advancing the Field}

\author{
Vijay A. Mittal \\ Departments of Psychology and Psychiatry, Institute for Policy Research, Medical Social Sciences, \\ Institute for Innovations in Developmental Sciences, Northwestern University, Evanston, IL, USA
}

Strik et al. [1] present the Systems Neuroscience of Psychosis (SyNoPsis) project, a conceptual framework that approaches schizophrenia as a disorder of interindividual communication. SyNoPsis seeks to circumvent issues faced by traditional strategies for categorizing and understanding etiology-symptom relationships by taking a consistent empirical approach to matching clinical manifestations of schizophrenia that (1) center around abnormalities in communication and (2) are closely tied to brain circuits relevant to motor, associative, and limbic functions that underlie human behavior. The SyNoPsis team has used this novel framework to guide an in-depth literature review and subsequently develop an innovative assessment scale strongly rooted in theory (Bern Psychopathology Scale [BPS]) [2]. Further, ongoing SyNoPsis studies have been applied to informing treatment in practical ways. For schizophrenia, this approach has several advantages. In addition, the ongoing effort has raised important questions that will fuel exciting new directions in years to come.

Rather than attempting to link brain dysfunction directly to a traditional psychiatric disorder (i.e., the classic and predominant approach to investigating etiology and treatment in psychiatry and psychology), the National Institute of Mental Health (NIMH) Research Domain Cri- teria $(\mathrm{RDoC})$ initiative relies on intermediate functional domains that occur on a continuum of normative behaviors (e.g., cognitive and positive valence systems, social processes); the initiative is centered around in-depth and multifaceted investigation into brain circuit-domain relationships. By design, the ultimate link between brain circuits and traditional symptoms of psychopathology is secondary and occurs when extreme variations of behavior on intermediary continuums ultimately contribute to more complex symptoms of psychiatric illness [3]. SyNoPsis is similar to RDoC in that it emphasizes the importance of brain function in understanding psychiatric illness, but rather than focusing on intermediary functional constructs, SyNoPsis dramatically redefines the classic clinical symptoms instead, focusing on specific communication and intentional behavior deficits/abnormalities central to psychosis. Those phenomena that have been tied directly to corticocortical and corticobasal circuits (based on the empirical literature) are heavily weighted in this framework. More plainly, while RDoC aims to link the brain to a series of intermediary domains that then comprise symptoms, SyNoPsis redefines symptoms instead, and focuses directly on the brain-communicationoriented symptom link.

\section{KARGER}

(c) 2017 S. Karger AG, Basel

E-Mail karger@karger.com

www.karger.com/nps
Vijay A. Mittal, PhD, Departments of Psychology and Psychiatry Institute for Policy Research, Medical Social Sciences Institute for Innovations in Developmental Sciences, Northwestern University Swift Hall 102, 2029 Sheridan Road, Evanston, IL 60208 (USA)

E-Mail vijay.mittal@ northwestern.edu 
There are several significant benefits to this approach. First, focusing on a direct brain-symptom link removes the necessity of intermediary symptoms; Strik et al. [1] note that with $\mathrm{RDoC}$, the causal gap between domains and symptoms may be too abstract. There is a risk that in adhering to $\mathrm{RDoC}$ the field may become adept at understanding underlying causes of the subordinate intermediary functions (i.e., RDoC domains/constructs), but ultimately, these individual units may not combine in a parsimonious fashion to explain clinical symptoms in their entirety. Second, researchers are seeking to find a reconciliation between the RDoC approach that sees symptoms of psychopathology as the amalgamation of extremes on one or more intermediate dimensions of normative functioning (e.g., the symptom anhedonia may reflect a low level of activity on reward-related constructs in the positive valence systems domain) and characteristics that may fall outside of a normative continuum (e.g., deviations of kind that are central to psychotic disorders, such as thought disorder and hallucinations) [4]. In contrast, SyNoPsis is rooted in a theoretical foundation that is specific to characteristics of schizophrenia, providing allowance for deviations of degree as well as deviations of kind. This provides a viable alternative or supplementary dimensional approach to investigating psychosis.

Outside of this comparison, SyNoPsis also provides several promising additional features. First, the system fits well with other compelling theories of psychosis. For example, the communication-centered focus of SyNoPsis shares components seen in other theoretical models [5], and furthermore the basal ganglia circuits in question are modulated by neurotransmitter systems critically implicated in leading theories of psychosis [6]. Together with the unique perspectives taken by SyNoPsis, this convergence will allow for a research agenda with significant relevance as well as a platform for investigations driven by competing hypotheses. Further, the system is centered on corticocortical and frontosubcortical circuits. If psychosis is a disorder that includes such a wide variety of symptoms and characteristics, circuits linking and modulating information between systems remain an excellent target. The frontal subcortical circuits in particular are promising targets, as they have been strongly implicated in psychosis and across the continuum [7-12] and they regulate core foundations of human behavior and dysfunction including cognitive, movement, and emotive functions. Finally, there is significant potential for SyNoPsis to directly contribute to practical improvements in treatment development. Specifically, the system highlights novel and important targets (e.g., gesture behavior), which drive so- cial and functional outcomes in schizophrenia [13-16]. Further, SyNoPsis provides a roadmap to address the language system deficits contributing to auditory hallucinations, and the group has been active in demonstrating the efficacy of this approach with brain stimulation [17]. In addition, SyNoPsis drives exploratory analyses of motor dysfunction, an area with limited treatment options, with the intention of generating novel interventions [18].

The SyNoPsis framework has also generated several important questions. The coming years will be an exciting time as the team continues to address these issues. First, it is important to consider that while SyNoPsis does include several components that are not found in $\mathrm{RDoC}$, it can still benefit from integration with or lessons learned from the RDoC system. For example, how will a system that is built around schizophrenia specifically be practically employed for understanding a shared and distinct phenomenology across serious mental illnesses (an important direction in our field), or, further, address comorbidity within schizophrenia? Second, RDoC places the brain circuits directly in the center of a units of analysis matrix (which ranges from genes all the way up to behavioral paradigms), and this, in part, stresses the contribution of building block components (e.g., genes, molecules, and cells) on one side, and further modulating influences between the brain and ultimate behavior (e.g., physiology) on the other. How will SyNoPsis continue to develop in a way that highlights the impact of important factors that contribute to brain circuit abnormalities or, further, moderate the relationship between the brain and symptomatology? It will be important for the SyNoPsis framework to integrate important factors that contribute to psychosis symptoms (genes, hormones, and physiology). On a related point, how will the system continue to develop to incorporate features that are not directly specific to the corticocortical and basal ganglia circuits or to communication, but are relevant to psychosis? For example, how would SyNoPsis integrate and consolidate cerebellar circuit dysfunction and cognitive dysmetria into the conceptual framework [19-22]? Another question relates to the emphasis on communication. One of the strengths of SyNoPsis is that it is strongly tied to a theoretical orientation, but this might be seen as a limiting factor as well. For example, are there other central aspects of schizophrenia that would have served as an organizing factor? As noted, the circuits in question influence a range of functions, and it may be challenging to attribute related dysfunctions to a single conceptual category. Given the strong theoretical underpinnings, it will also be important for SyNoPsis to continue to develop 
and adapt, incorporating incoming findings that support alternative viewpoints and avoiding the trap of primarily driving confirmatory research. I believe that answering these questions will simultaneously improve SyNoPsis and advance our field.

In conclusion, SyNoPsis shows enormous potential for guiding theoretically informed research in a manner that is designed to directly inform intervention development. The BPS is an exceptional instrument, and I look forward to using it, as well as the broader SyNoPsis orientation, to help devise my research program. I will be particularly excited to see this system employed to understand and guide work around pressing issues in our field, such as human development, early intervention and identifica- tion (prodromal syndromes), questions around subtyping and generalized versus specific deficits, and individualized medicine/targeted treatment outcomes. This is an exciting time for psychosis research, and dynamic and theoretically founded systems like SyNoPsis will continue to make a large and critical impact.

\section{Disclosure Statement}

Dr. Mittal is a consultant for Takeda. There are no disclosures or conflicts of interests. This work was supported in part by the NationalInstitutesofHealth(RO1MH094650,R21/R33MH103231, and R21MH110374 to V.A.M.).

\section{References}

1 Strik W, Stegmayer K, Walther S, Dierks T: Systems Neuroscience of Psychosis: mapping schizophrenia symptoms onto brain systems. Neuropsychobiology 2017;75:100-116.

2 Strik W, Wopfner A, Horn H, Koschorke P, Razavi N, Walther S, Wirtz G: The Bern Psychopathology Scale for the assessment of system-specific psychotic symptoms. Neuropsychobiology 2010;61:197-209.

3 Insel T, Cuthbert B, Garvey M, Heinssen R, Pine DS, Quinn K, Sanislow C, Wang P: Research Domain Criteria (RDoC): toward a new classification framework for research on mental disorders. Am J Psychiatry 2010;167: 748-751.

4 Mittal VA, Wakschlag LS: Research Domain Criteria (RDoC) grows up: strengthening neurodevelopment investigation within the RDoC framework. J Affect Disord 2017;216: 30-35.

5 Crow TJ: Is schizophrenia the price that Homo sapiens pays for language? Schizophr Res 1997;28:127-141.

6 Howes OD, Kapur S: The dopamine hypothesis of schizophrenia: version III - the final common pathway. Schizophr Bull 2009;35: 549-562.

7 Graybiel AM: The basal ganglia and cognitive pattern generators. Schizophr Bull 1997;23: 459-469.

8 Robbins TW: The case for frontostriatal dysfunction in schizophrenia. Schizophr Bull 1990;16:391-402.
9 Mittal VA, Jalbrzikowski M, Daley M, Roman C, Bearden CE, Cannon TD: Abnormal movements are associated with poor psychosocial functioning in adolescents at high risk for psychosis. Schizophr Res 2011;130:164169.

10 Mittal VA, Dean DJ, Pelletier A, Caligiuri M: Associations between spontaneous movement abnormalities and psychotic-like experiences in the general population. Schizophr Res 2011;132:194-196.

11 Mittal VA: Cross-cutting advancements usher in a new era for motor research in psychosis. Schizophr Bull 2016;42:1322-1325.

12 Mittal, VA, Bernard JA, Northoff G: What can different motor circuits tell us about psychosis? An RDoC perspective. Schizophr Bull 2017;43:949-955.

13 Walther S, Mittal VA: Why we should take a closer look at gestures. Schizophr Bull 2016; 42:259-261.

14 Bernard JA, Mittal VA: Updating the research domain criteria: the utility of a motor dimension. Psychol Med 2015;45:2685-2689.

15 Walther S, Eisenhardt S, Bohlhalter S, Vanbellingen T, Müri R, Strik W, Stegmayer K: Gesture performance in schizophrenia predicts functional outcome after 6 months. Schizophr Bull 2016;42:1326-1333.

16 Osborne KJ, Bernard JA, Gupta T, Dean DJ, Millman Z, Vargas T, Ristanovic I, Schiffman J, Mittal VA: Beat gestures and postural control in youth at ultrahigh risk for psychosis. Schizophr Res 2017;185:197-199.
17 Homan P, Kindler J, Federspiel A, Flury R, Hubl D, Hauf M, Dierks T: Muting the voice: a case of arterial spin labeling-monitored transcranial direct current stimulation treatment of auditory verbal hallucinations. Am J Psychiatry 2011;168:853-854.

18 Walther S, Schäppi L, Federspiel A, Bohlhalter S, Wiest R, Strik W, Stegmayer K: Restingstate hyperperfusion of the supplementary motor area in catatonia. Schizophr Bull 2017; 43:972-981.

19 Bernard JA, Mittal VA: Dysfunctional activation of the cerebellum in schizophrenia: a functional neuroimaging meta-analysis. Clin Psychol Sci 2015;3:545-566.

20 Andreasen NC, Paradiso S, O'Leary D: “Cognitive dysmetria" as an integrative theory of schizophrenia: a dysfunction in cortical-subcortical-cerebellar circuitry? Schizophr Bull 1998;24:203-218.

21 Dean DJ, Kent JS, Bernard JA, Orr JM, Gupta T, Pelletier-Baldelli A, Carol EE, Mittal VA: Increased postural sway predicts negative symptom progression in youth at ultrahigh risk for psychosis. Schizophr Res 2015;162: 86-89.

22 Dean DJ, Bernard JA, Orr JM, PelletierBaldelli A, Gupta T, Carol EE, Mittal VA: Cerebellar morphology and procedural learning impairment in neuroleptic-naive youth at ultrahigh risk of psychosis. Clin Psychol Sci 2014;2:152-164.
SyNoPsis Provides a Promising Framework for Advancing the Field 